\title{
Incorporation mystique et subjectivité féminine d'après le Livre d'Angèle de Foligno († 1309)
}

\section{Damien Boquet}

\section{OpenEdition}

1 Journals

\section{Édition électronique}

URL : https://journals.openedition.org/clio/6553

DOI : $10.4000 /$ clio.6553

ISSN : 1777-5299

Éditeur

Belin

\section{Édition imprimée}

Date de publication : 1 novembre 2007

ISBN : 978-2-85816-940-5

ISSN : $1252-7017$

\section{Référence électronique}

Damien Boquet, «Incorporation mystique et subjectivité féminine d'après le Livre d'Angèle de Foligno († 1309) », Clio. Femmes, Genre, Histoire [En ligne], 26 | 2007, mis en ligne le 23 août 2013, consulté le 23 avril 2022. URL : http://journals.openedition.org/clio/6553; DOI : https://doi.org/10.4000/clio.6553

Ce document a été généré automatiquement le 23 avril 2022.

Tous droits réservés 


\title{
Incorporation mystique et subjectivité féminine d'après le Livre d'Angèle de Foligno († 1309)
}

\author{
Damien Boquet
}

«...la prophétie a été transférée au sexe

féminin. »

Les Instructions d'Angèle de Foligno

1 Au XIII siècle, une nouvelle expression de la piété se diffuse dans la littérature religieuse : le "mysticisme féminin ». Qu'elles soient prophétesses, visionnaires ou dévotes transportées d'extase, ces femmes mystiques ont toutes en commun une relation privilégiée avec Dieu, plus précisément avec son incarnation, Jésus. Celui-ci les a choisies entre mille, pour se montrer à elles, pour leur parler, pour leur révéler intimement ses mystères. En réponse à cette élection, elles offrent au Christ tout leur amour et toute leur compassion dans une exclusivité qui se veut fusionnelle. La civilisation médiévale, normée par les hommes, a assigné à la femme une identité modelée par les affects et les émotions. La mystique féminine de la fin du Moyen Âge peut apparaître comme un avatar de cette nature façonnée par la domination masculine. Elle émerge à une époque où triomphe le pouvoir institutionnel de l'Église qui s'est imposée en médiatrice indispensable entre Dieu et les hommes. Jamais le groupe des clercs n'avait été en position d'encadrer aussi étroitement les pratiques et les croyances religieuses des populations laïques qui, de leur côté, sont en demande d'une participation plus étroite au sacré. Parmi les fidèles, les exigences des femmes sont sans doute plus fortes, parce qu'elles sont de facto exclues du sacerdoce ; peut-être aussi parce que le cadre monastique qu'on leur propose est saturé et souffre d'une discipline relâchée. C'est dans ce contexte qu'on voit se multiplier les récits sur des laïques - dont certaines se font recluses, béguines ou tertiaires - qui entretiennent un dialogue intime avec Jésus.

2 Pour le lecteur d'aujourd'hui, cette littérature mystique, ponctuée d'extases et autres étreintes enflammées, peut paraître irrationnelle, à tout le moins déconcertante. Que 
penser de ces femmes qui affirment voir régulièrement le Christ en pensée ou bien même physiquement, qui lui parlent et à qui il répond ? Que dire de celles qui défaillent à la seule vue de l'hostie, de celles encore qui se laissent mourir de faim ou bien se mutilent pour vivre dans leur chair le supplice de la crucifixion? Inévitablement, le lecteur contemporain sera tenté de voir dans ces manifestations, que Michel de Certeau qualifie d'» extraordinaire psychosomatique $»^{1}$, des symptômes, ceux de la pathologie mentale, de l'hallucination ou de l'autosuggestion hystérique ${ }^{2}$. En tout cas, le sentiment qui s'impose est celui d'une profonde étrangeté avec une telle sensibilité.

Parmi les expressions les plus radicales de cette mystique féminine, il y a les phénomènes d'incorporation. J'entends par là l'évocation de motifs de piété où il est question soit d'insertion, physique ou spirituelle, de tout ou partie du corps du Christ, ou encore des instruments de sa passion, dans le corps ou l'âme de la mystique ; soit la réciproque, c'est-à-dire l'insertion de la mystique elle-même dans le corps du Christ. Mon objectif est de montrer que, loin de tout essentialisme féminin ou du réductionnisme qu'impose une vision "clinicienne" de la mystique ${ }^{3}$, ces motifs d'incorporation, si fréquents dans les témoignages sur la piété des femmes à la fin du Moyen Âge, participent d'une culture mixte et non marginale qui doit être mise en relation avec les discours savants des théologiens. Ce faisant, je plaide pour une histoire équilibrée de la mystique féminine, qui tienne simultanément compte des apports d'une anthropologie historique des relations hommes/femmes et d'une histoire intellectuelle de la pensée religieuse.

4 Pour mener l'exploration, j'ai isolé parmi bien d'autres un dossier, celui de la mystique italienne Angèle de Foligno. Angèle naît vers 1248 à Foligno, en Ombrie dans la patrie de François d'Assise. Elle connaît une première vie d'épouse et de mère sur laquelle on ne sait rien, si ce n'est que mari et enfants ne sont plus en vie quand elle choisit d'entrer dans le tiers ordre franciscain en 1291. Elle continue de vivre chez elle en recluse, jusqu'à sa mort en 1309, tout en exerçant une influence sur un petit groupe de disciples. Ces quelques données sont essentielles. Notamment parce qu'elles établissent Angèle dans la longue lignée des femmes laïques profondément marquées par la figure de François d'Assise, comme ses contemporaines Douceline de Provence ( $\dagger$ 1274) ou Marguerite de Cortone ( $†$ 1297). La perfection féminine promue par les milieux de la curie pontificale est alors prioritairement franciscaine : la moitié des dix femmes ayant fait l'objet d'un procès de canonisation entre 1198 et 1431 était liée au mouvement franciscain ${ }^{4}$. Néanmoins, le cas d'Angèle est plus sensible puisqu'elle fut proche d'Ubertin de Casale - qui reconnaîtra avoir été transformé par sa rencontre avec la Folignate -, un des chefs de file du mouvement dissident des Spirituels, partisans d'une pauvreté radicale de l'ordre, contre qui la répression pontificale s'intensifie dans la décennie qui suit la mort d'Angèle. Faut-il voir alors dans l'enseignement de celle-ci une parenté avec d'autres textes spirituels, notamment avec l'Arbre de la vie d'Ubertin? La question demeure posée ${ }^{5}$.

5 On possède le récit des expériences mystiques et de l'enseignement d'Angèle à travers deux textes, traditionnellement appelés le Mémorial et les Instructions, qui rassemblés constituent le Livre 6 . La genèse de ces deux textes s'avère particulièrement compliquée, comme c'est souvent le cas d'ailleurs pour ces témoignages autour des expériences mystiques féminines ${ }^{7}$. Angèle elle-même ne savait sans doute ni lire ni écrire, en tout cas pas le latin. Elle n'est donc pas à proprement parler l'auteur des textes qui transcrivent son expérience, souvent à la première personne. C'est ainsi que le Mémorial 
est présenté et rédigé par son confesseur et parent, un frère franciscain. Quant aux Instructions, leur structure est encore plus complexe, puisqu'elles ont été composées par plusieurs mains, et en partie au moins après la mort d'Angèle ${ }^{8}$. Il ne faut pas imaginer derrière Angèle une stricte individualité, mais plutôt comme l'écrit A. Boureau un " entrelacs de voix », un « compromis textuel entre une aspiration mystique féminine, les exigences religieuses du parti spirituel franciscain et le contrôle clérical » ${ }^{9}$ La subjectivité religieuse que nous allons tenter de dégager n'est donc pas celle d'une individualité exclusive mais celle d'une figure idéale produite collectivement, au féminin et au masculin, entre la fin du XIII ${ }^{e}$ siècle et le début du XIV ${ }^{e}$ siècle.

6 Trois motifs d'incorporation - autour de l'eucharistie, des plaies du Christ et des instruments de la Passion - seront successivement analysés au regard de leur tradition spirituelle, avant d'être repris sous l'angle d'une appréhension de la figure d'Angèle comme sujet incorporé.

\section{Incorporation eucharistique}

7 Depuis les premiers siècles du christianisme, la commémoration de la Cène est le symbole sacré par excellence, le sacrement, mais il faut attendre le XIII ${ }^{e}$ siècle pour que le rituel de l'eucharistie entre véritablement dans la vie quotidienne des fidèles. Pendant des siècles, la communion demeure une pratique exceptionnelle pour les laïcs et l'eucharistie est envisagée par les théologiens essentiellement sous l'angle doctrinal. Ainsi, entre le $I X^{\mathrm{e}}$ et le $X I I^{\mathrm{e}}$ siècle, les débats sont vifs entre théologiens sur la question de la nature de la présence du Christ au cours du rituel. A la fin $\mathrm{du} \mathrm{XI}^{\mathrm{e}}$ siècle, les partisans d'une présence réelle du Christ dans l'hostie consacrée l'emportent au sein de l'Église. En 1215, le concile de Latran IV rend obligatoire la communion annuelle: l'eucharistie, avec la confession, devient l'outil privilégié d'encadrement religieux des populations. Très vite un véritable culte eucharistique se développe, favorisé par la pratique nouvelle de l'élévation lors de la messe puis par l'institution de la Fête-Dieu en 1264, notamment grâce à l'action d'une mystique du diocèse de Liège, Julienne de Cornillon $(\dagger 1258)^{10}$.

L'essor de la dévotion eucharistique témoigne donc d'une parfaite rencontre entre une stratégie pastorale et une attente des populations. Le dogme défendu et popularisé de la présence réelle dans la célébration eucharistique permet au fidèle d'établir une communication personnelle avec le Christ qui est directement accessible aux sens. Certaines femmes, comme Angèle, investissent alors fiévreusement la dévotion eucharistique. Le Livre fourmille d'évocations dans lesquelles le mystère de l'incorporation réelle, censé se révéler dans la transsubstantiation, est vécu de façon réaliste. L'incorporation devient littérale. Cette immédiateté physique de la présence réelle a pour premier effet d'engendrer une dépendance à l'hostie et à la communion. Angèle a faim d'hostie en permanence. Les situations décrites sont celles d'une addiction. Entre chaque communion, elle dit souffrir du manque (295) ; à l'inverse, la seule vue de l'hostie déclenche en elle des extases et des apparitions surnaturelles (66, $97,166,252)$. En outre, elle conçoit l'acte de communier très concrètement comme une ingestion du corps de Jésus, se livrant à ce que Caroline Bynum appelle un "cannibalisme symbolique ${ }^{11}:$ «... quand elle communie, l'hostie se répand dans sa bouche. Elle dit qu'elle n'a la saveur ni du pain ni d'aucune chair que nous connaissons. Certes, elle a bien une saveur de chair, mais une saveur très différente et très 
savoureuse » (135). Cette sensation de manger la chair du Christ, d'en ressentir la saveur, la texture, comme celle du sang aussi, se retrouvera chez d'autres mystiques postérieures, la plus célèbre étant Catherine de Sienne $(\dagger 1380){ }^{12}$. On assiste même chez Angèle à une forme de contamination de ces pratiques de dévotion dans des actes non liturgiques, comme le soin aux malades. Inspirée sans doute par le baiser au lépreux de saint François, un jour qu'elle soigne un lépreux, elle boit l'eau qui a servi à le laver et avale alors un lambeau de chair malade : " une petite croûte des plaies du lépreux était restée coincée dans ma gorge, et je m'efforçais de l'avaler. Ma conscience m'interdisait de la recracher, comme si j'avais communié, encore que je ne voulais pas la cracher pour la rejeter mais pour la détacher de ma gorge. » (105). Le dogme de la présence réelle pose en effet de multiples problèmes comme celui de la régurgitation de l'hostie. Angèle le sait, elle qui évoque ailleurs sa crainte qu'un morceau de l'hostie ne lui reste coincé entre les dents (135). Puisque l'hostie est vivante, on ne sera pas étonné qu'elle parle à Angèle, ou plutôt qu'à travers elle, au moment de l'élévation, lui parlent soit le Christ, soit Marie $(97,114,166)$. Il lui arrive de voir directement le Christ enfant dans l'hostie ou encore les yeux de Jésus, qui font éclipse puisqu'ils sont si gros, dit-elle, qu'on ne distingue plus que les bords de l'hostie (83). Ces apparitions sont si fréquentes que son confesseur prend l'habitude de lui demander au moment de la communion ce qu'elle voit $(98,134)$.

9 Il serait aisé de s'en tenir à une explication strictement psycho-pathologique de ces impressions physiques éprouvées par la recluse. Emportée par sa foi, Angèle semble bien avoir des hallucinations visuelles et auditives au moment de communier. Néanmoins, il convient aussi de replacer ces situations dans leur cohérence culturelle. En raison des exclusions et des relégations qui pèsent sur elles - exclusion du sacerdoce, relégation de la culture savante - les femmes trouvent une reconnaissance/ résistance/échappatoire dans une piété affective et peu intellectualisée qui valorise les espaces que la société masculine leur laisse. En particulier, il faut rattacher la dévotion eucharistique à la relation spécifique que la norme sociale pose entre la femme et la nourriture. La privation ascétique de nourriture et sa sublimation dans le festin mystique de l'eucharistie sont des manifestations très fréquentes du mysticisme féminin. Angèle n'échappe pas à la tentation de cette "anorexie sainte " ${ }^{13}$ : elle sent que moins elle mange, plus elle est en communion intense avec Dieu (62). En outre, il y a sous ces pratiques un enchevêtrement de références, hagiographiques, spirituelles voire théologiques dont il faut tenir compte en ce qu'elles structurent l'expérience et l'intègrent socialement. Angèle est un exemple assez précoce, mais peut-être est-elle déjà influencée par des modèles de piété féminine que les clercs ne manquent pas de présenter aux mulieres religiosae.

10 On doit penser ici à la très célèbre Vie de Marie d’Oignies († 1213) écrite peu après sa mort par son confesseur Jacques de Vitry. Marie, elle aussi, est tenaillée par la faim de la chair et la soif du sang sacrés : «Pour elle, vivre et prendre le corps du Christ, c'était la même chose $»^{14}$. Jacques cite dans la foulée les versets de Jean 6, 53-54: « Si vous ne mangez pas la chair du fils de l'homme et ne buvez son sang, vous n'aurez pas la vie en vous. Qui mange ma chair et boit mon sang a la vie éternelle », et déjà plus haut cet autre verset biblique : « ceux qui me mangent auront encore faim, ceux qui me boivent auront encore soif» $(\mathrm{Si} 24,21)^{15}$. C'est pourquoi, fort d'une lecture littérale de l'Écriture, Marie a le désir de ne se nourrir que d'hostie, à laquelle elle attribue une saveur de miel ; c'est pourquoi aussi elle n'éprouve aucune satiété ${ }^{16}$. Il lui arrive même de se faire saigner en avalant des morceaux de pain dur qui lui lacèrent la gorge comme 
succédanés d'une communion qu'elle trouve trop rare ${ }^{17}$. Évoquons également l'influence du discours ascétique qui vient appuyer la théologie de la présence réelle. Depuis le XIe siècle, le dévot qui s'offre par son ascèse en sacrifice est comparé dans les écrits spirituels à une "hostie vivante " (hostia viva) ${ }^{18}$, selon la parole de saint Paul (Rom., 12, 1), par analogie avec le sacrifice du Christ ${ }^{19}$. Bientôt cette hostia viva devient proprement animée. On le constate par exemple à l'évocation d'un autre modèle, celuilà assurément connu et médité par Angèle : Claire d'Assise ( $† 1253)$ dont la Vie relate qu'un jour de 1240 où Frédéric II avait lancé ses mercenaires à l'assaut d'Assise, elle s'adressa directement à l'hostie consacrée pour la supplier de protéger son monastère et d'épargner ses sœurs. Le texte rapporte qu'une voix d'enfant s'éleva de la cassette contenant l'hostie pour rassurer les saintes femmes ${ }^{20}$. On observe alors la même association, chez Angèle comme chez Claire, de l'eucharistie avec la figure du Christ enfant $^{21}$; ce qui n'est guère surprenant quand on sait que cette même dévotion à l'enfant Jésus a été l'un des premiers motifs façonnés par la spiritualité monastique réformée - cistercienne en l'occurrence avec notamment le traité d'Aelred de Rievaulx († 1167) Quand Jésus eut douze ans - à avoir été investis par la piété féminine, comme le montre l'exemple précoce de Christine de Markyate ( $†$ c.1160 ${ }^{22}$.

Des témoignages d'incorporation eucharistique rencontrés dans le Livre, on retiendra qu'ils s'inscrivent dans une généalogie spirituelle et une exemplarité hagiographique déjà anciennes, puisqu'il est aisé d'en remonter le fil sur près de deux siècles, mais surtout dans cette tendance forte de la mystique féminine à reprendre de façon littérale des motifs émanant des milieux monastiques réformés, principalement cisterciens.

\section{Incorporation aux plaies du crucifié}

La dévotion aux plaies du crucifié participe aussi de cette évolution au XIII siècle qui consiste à voir dans la Passion l'événement majeur de la rédemption. L'institution ecclésiastique apporte sa pierre à l'édifice en établissant une fête pour la plaie du côté avant que ne se mette en place au XIV siècle le très populaire culte au Sacré Cœur. C'est l'époque aussi où se répand le miracle de la stigmatisation, marque suprême de l'élection divine offerte à celui qui a poussé à l'extrême le désir et la pratique de conformation au Christ souffrant. Or, mis à part la célèbre exception du précurseur François d'Assise, la stigmatisation au Moyen Âge est un miracle féminin. Angèle ne reçoit pas les stigmates. Néanmoins, elle voue un culte très assidu aux plaies qui la conduit progressivement vers l'incorporation au crucifié. Il y a comme un premier stade, où une certaine distance est encore maintenue, lorsque le Christ l'invite à plonger son regard dans ses plaies (57). C'est déjà pour elle un moment de grande jouissance que de contempler les marques des clous (121). Mais ce qu'elle attend, c'est le contact, pleinement sanctifiant (116), qui s'achève dans l'étreinte: "et alors il m'appela et me dit de poser ma bouche sur la plaie de son côté » (58). Ce "bouche à plaie" construit immédiatement un canal mystique par lequel la science divine est infusée dans la mystique (58). Enfin, il y a le dernier stade de l'incorporation totale: " mon âme a parfois l'impression qu'elle pénètre dans le côté du Christ, avec grande joie et plaisir. » (121). L'analogie entre l'incorporation mystique dans les plaies et le culte eucharistique est favorisée par la référence commune au sang ${ }^{23}$, le sang qui s'écoule des plaies et que la dévote est invitée à boire : «j'eus l'impression de voir et de 
boire son sang en train de couler de son côté, et il m'était donné de comprendre qu'en ce sang il me purifiait » (58). Une vision montre même les compagnons d'Angèle avec les lèvres voire tout le visage couvert de sang après qu'ils les ont plongés dans la plaie du côté (215). À la fin du Livre, Angèle à l'article de la mort se voit tout entière immergée dans le sang du Christ : «ce sang était si frais et si chaud, comme s'il coulait du corps du crucifié. Il fut dit alors à son âme : voici ce qui t'a purifiée » (298).

Une fois encore, il convient d'aborder avec prudence ces images de pénétration avec épanchement de fluides, serties dans une rhétorique amoureuse explicite, qui deviendront une des marques distinctives de la mystique féminine de la fin du Moyen Âge. D'abord parce que ces motifs sont apparus aux XI ${ }^{\mathrm{e}}-\mathrm{XII} \mathrm{I}^{\mathrm{e}}$ siècles, sous la plume d'auteurs monastiques masculins, des abbés qui écrivaient principalement pour un public masculin, leurs compagnons de cloître. Dans cette rhétorique une place essentielle est évidemment dévolue au rapport masculin/féminin mais ne se traduit pas simplement par une césure homme/femme ${ }^{24}$. Surtout, il ne faudrait pas oublier que ces images possèdent une origine exégétique, une généalogie intellectuelle qui s'est construite bien en amont des spécificités socio-culturelles de la mystique des femmes laïques de la fin du Moyen Âge. En ce sens, le motif de l'incorporation aux plaies du Christ se rencontre déjà chez Bernard de Clairvaux ( $† 1153$ ) (qui s’inspire lui-même des Pères de l'Église ${ }^{25}$ dans un commentaire d'un verset du Cantique des cantiques : « Ma colombe, cachée au creux des rochers,/ en des retraites escarpées,/ montre-moi ton visage » (Cant., 2, 14). Bernard propose comme interprétation : « Si [l'Église] entend ces paroles "ma colombe dans les trous du rocher", c'est qu'elle se tient dans les blessures du Christ avec toute sa ferveur et y demeure par une méditation continuelle $»^{26}$. À la même époque précisément, un autre abbé cistercien, Guerric d'Igny ( $† 1157$ ), fait la même lecture de Cant., 2, 14 et la prolonge, dans un sermon adressé à ses moines, en façonnant ce motif de l'incorporation mystique: «la raison pour laquelle le Christ tendre et miséricordieux a ouvert son côté, c'est que le sang de la plaie te vivifiât, que la chaleur de son corps te réchauffât, que l'aspiration de son cœur t'attirât pour ainsi dire par cet orifice librement ouvert ${ }^{27}$. Enfin, Aelred de Rievaulx dans la Vie de recluse confère à l'image une connotation plus affective et fusionnelle encore, dans un contexte cette fois ouvertement lié à une spiritualité féminine : «Vraiment, des fleuves ont jailli pour toi de la pierre, des trous ont été percés dans la muraille de son corps : les blessures de ses membres, aux creux desquelles, comme la colombe, tu peux t'aller blottir, les baisant une à une. Et le sang sur tes lèvres tracera un fil d'écarlate, et ta parole deviendra suave $»^{28}$. En suivant brièvement la seule veine cistercienne, on voit clairement comment au milieu du XII siècle, dans un même environnement culturel, une exégèse très ancienne d'un verset du Cantique des cantiques se transforme en un motif de piété faisant appel à l'imagination et à l'affect.

\section{Incorporation des instruments de la Passion}

Dernier motif d'incorporation étudié, la dévotion à la croix et aux outils de la Passion est aussi très ancienne. Des fêtes liturgiques consacrées à la croix existent depuis le VIe siècle à Rome. Quant au culte des reliques de la vraie croix, il est attesté en Occident dès la fin du VIIIe siècle. À partir de la fin du Xe siècle, la dévotion à la croix est encouragée notamment par la liturgie de Cluny; il devient alors fréquent de placer un crucifix à l'intérieur des églises. Plus tard, au XIIIe siècle, le recueil hagiographique de Jacques de 
Voragine La Légende dorée va aider à diffuser le culte de la sainte croix ${ }^{29}$. Si, à l'origine, la croix symbolisait le Christ triomphant, au XIII ${ }^{e}$ siècle, sous l'influence de la dévotion à la Passion, le crucifié exposé à la méditation des fidèles est le Christ souffrant ${ }^{30}$. C'est ce type de représentation qu'Angèle a sous les yeux lorsqu'elle se place, dit le rédacteur du Mémorial, au plus près de la croix dans l'église $(113,120)$, aux côtés du crucifié qu'elle appelle le "Dieu-homme souffrant» (121). La dévotion au crucifié est devenue dans le courant du XII ${ }^{e}$ siècle l'un des thèmes majeurs de la spiritualité monastique, là encore sous l'influence principale des moines réformés ${ }^{31}$. C'est désormais la souffrance du crucifié qui condense l'acte sacrificiel du Dieu-homme et la rédemption de l'humanité. L'imitation du Christ se fait alors par compassion et identification à ses souffrances sur la croix. Sans multiplier les exemples, on peut revenir au témoignage de Guerric d'Igny. Ainsi, dans un de ses sermons pour le dimanche des Rameaux consacré à la «mémoire de la croix ", il incite chaque moine à rendre grâce en se crucifiant à son tour par l'ascèse. Les images se font franchement réalistes : «La crainte de Dieu, en effet, nous fixe à la croix comme feraient des clous solidement plantés ${ }^{32}$. Il rappelle la parole de Paul qui dit porter en son corps les «stigmates de Jésus » (Gal., 6, 17), devenue sous la plume de Guerric les "stigmates du crucifié » ${ }^{33}$. Bientôt, il franchit le pas de l'identification en appelant Paul «celui qui fut crucifié avec le Christ ${ }^{34}$. Tout le motif d'une incorporation symbolique de la crucifixion est contenu dans la péroraison du sermon : "Tu peux aller en toute sécurité, en toute confiance, là où ton juge est ton avocat : la seule condition, c'est que ton esprit emporte avec soi le symbole de la croix ${ }^{35}$, la mortification de Jésus que tu portes partout et toujours en ton corps " ${ }^{36}$. C'est à cette source que vient puiser la rhétorique mystique d'Angèle.

On trouve ainsi chez Angèle de multiples références à la croix comme symbole de la douleur et du sacrifice du Christ pour le salut des hommes. Selon un procédé récurrent, Angèle rapporte alors ce sacrifice à elle-même. Elle se considère responsable de la crucifixion : « je sentais que je l'avais moi-même crucifié » (55). Dès lors, la seule voie de conformation au Christ est de s'offrir elle-même en sacrifice, en revendiquant sa propre crucifixion : « je lui demandais [au Christ] de me faire cette grâce : puisque le Christ fut crucifié sur le bois, qu'il me crucifie dans une ravine, ou en un lieu très abject, ou sur une chose très abjecte» (59). L'auto-crucifixion est d'abord un acte de pénitence. Angèle appelle ainsi "travaux de la croix » (194) ou ailleurs "discipline de la croix » (243) cet itinéraire de pénitence: "il est bon que chacun s'efforce le plus qu'il peut d'être cloué avec le crucifié béni » (216). De la douleur de contrition, on glisse vers une douleur de compassion (61), qui est dans le même temps plaisir de la participation voire de la fusion. La croix change alors de nature ; d'instrument de torture, elle devient lieu de repos $(161-2,170,195,285)$ et même de jouissance : « la croix est ton salut et ton lit, et elle doit être ton plaisir, car elle est ton salut »(103).

Enfin, on retrouve la métaphore de l'incorporation. D'abord concernant le Christ luimême qui a la croix et les instruments de son supplice incrustés, dit Angèle, dans son âme et dans son corps (162). Ses blessures sont même la preuve à la fois de sa toutepuissance et de sa volonté d'être totalement homme puisqu'il a lui-même donné pouvoir aux épines, aux clous comme à la lance de le transpercer (255-6). Ailleurs, c'est à l'inverse la chair du Christ qui s'incruste dans la croix, en sanctifiant alors la matière inerte : « une fois, je pensais à la grande douleur que le Christ supporta en croix et je pensais à ces clous ; j'avais entendu dire que ces clous avaient emporté la chair de ses mains et de ses pieds au-dedans du bois ${ }^{37}$. Je désirais voir au moins un peu de la chair 
du Christ que les clous avaient emportée dans le bois " (81). Ce peut être enfin Angèle qui incorpore la croix en elle : " aussitôt je ressentais cette croix et cet amour tout audedans de mon âme. Je ressentais cette croix corporellement, et en la ressentant, mon âme se liquéfiait dans l'amour de Dieu » (78). Comment ne pas penser, à l'évocation de cette ultime sensation d'incorporation, au cas exemplaire de la religieuse Claire de Montefalco $(\dagger 1308)$ dont le cœur fut disséqué à sa mort et qui portait gravé en miniature, au dire de ses sœurs, non seulement l'image du Christ crucifié mais aussi les instruments de la Passion?

\section{Le sujet incorporé}

Comment évaluer ces trois motifs d'incorporation mystique dans la perspective d'une anthropologie historique de la subjectivité ? Le premier constat concerne la perception du corps dans sa matérialité, le sien propre ou celui du Christ. Angèle conçoit le corps moins comme une totalité que comme une sorte de mécano, un agrégat de fragments. Le Christ qui se révèle à elle passe en revue tous ses membres suppliciés (149) : ses mains et ses pieds transpercés mais aussi son cou (82). Il expose même à son adoration les poils qu'on lui a arrachés, ceux de sa barbe et des sourcils ou encore ses cheveux (57). Cette perception fragmentaire du corps est sans doute favorisée par la dévotion aux plaies du Christ ${ }^{38}$ et par le culte des reliques qui confère une efficacité sacrée, donc une vie propre et autonome, à des portions imputrescibles du corps, os ou cheveux. À partir de là, comment se fait l'agencement de la machine corporelle? Guillemette Bolens a montré que deux logiques principales assurent dans la pensée occidentale la cohésion du corps perçu et décrit : la logique du corps-enveloppe et celle du corpsarticulaire $^{39}$. La conception dominante est celle du corps-enveloppe; elle s'organise autour de la distinction entre l'externe et l'interne, les orifices jouant le rôle de seuil entre l'individu et le monde. Cette perception s'accorde parfaitement avec la dévotion aux plaies. De façon plus surprenante, la lecture articulaire du corps tient une place importante dans le Livre, que ce soit pour traduire la douleur qui étreint Angèle dans toutes ses articulations $(153,183)$ jusqu'à la dislocation (77) ou pour exprimer son plaisir lors la communion eucharistique (98). Cette logique de la perception est encore plus flagrante lorsque est décrit le corps supplicié de Jésus: «il semblait aussi que toutes les articulations de ce corps béni étaient si disjointes, disloquées et désunies, à cause de la cruelle tension et de l'horrible traction infligées à ses membres virginaux sur le gibet de la croix par les mains homicides et perfides. Les tendons et les jointures des os de ce corps très sacré semblaient avoir totalement quitté leur harmonie normale; la peau ne représentait toutefois aucune solution de continuité » (214). Angèle précise bien que la peau n'était pas déchirée car l'orthodoxie doctrinale lui interdit de percer de nouveaux orifices; en revanche, rien ne l'empêche d'investir le terrain, neutre sur le plan théologique, des traumatismes internes. On voit de nouveau comment une sensibilité religieuse s'accorde et s'adapte à partir d'un cadre théologique prédéfini, sans oublier là encore l'impact du culte des reliques qui rend familière la dissémination des squelettes désarticulés.

18 Assurément, cette appréhension composite et fragmentée du corps brouille les frontières traditionnelles du sujet chrétien. On sait que l'anthropologie chrétienne depuis saint Paul, et sous l'influence du néo-platonisme, oppose fortement le corps et l'esprit. Le corps abrite le péché tandis que l'esprit est ce par quoi l'homme est à la 
ressemblance de Dieu. Au XIII ${ }^{e}$ siècle, cette approche dialectique a partiellement volé en éclats sous l'effet de la scolastique d'inspiration aristotélicienne, de l'émergence d'une science de la nature ou encore de spiritualités centrées sur l'incarnation. Sans doute faudrait-il aussi compter avec l'obligation pour l'Église de répondre au formidable défi lancé par les dualismes hétérodoxes. Les sens, la matière, la chair peuvent désormais avoir leur rôle à jouer dans l'économie du salut. Chez les femmes mystiques, cet infléchissement des discours et des mentalités se manifeste dans la place dévolue aux sens lors les épisodes extatiques. Dans le cas d'Angèle, l'événement mystique (vision, ressenti compassionnel, extase) est le plus souvent déclenché par une perception visuelle. L'église en est le cadre privilégié. Angèle est très sensible au décor : sa servante Masazuola est ainsi obligée de masquer les fresques de la Passion tant leur vue la fait horriblement souffrir (63). À Assise, c'est un regard porté sur un vitrail de la basilique qui déclenche la manifestation surnaturelle (77). Plus fréquemment, celle-ci survient quand elle regarde le crucifix ou lors de l'élévation de l'hostie. Perception sensorielle et fabrication d'images mentales sont donc les facultés mobilisées dans cette mystique imaginative : « les paroles humaines sont impuissantes à exprimer ce qui est raconté cidessous et qui s'est passé dans son corps ou dans son imagination » (213). A n'en pas douter, les auteurs qui transcrivent l'expérience d'Angèle savent parfaitement actionner les rouages de l'anthropologie savante, celle par exemple élaborée par les victorins et les cisterciens au XII ${ }^{e}$ siècle qui fait de l'imagination et de la sensation les deux facultés, l'une spirituelle, l'autre corporelle, capables d'assurer l'unité du composé humain en liant l'âme et le corps ${ }^{40}$. L'in-habitation surnaturelle d'Angèle obéit à un mécanisme anthropologique précis : elle opère par cet interstice entre le corps et l'âme qui fait de la mystique un être ouvert. En ce sens, on est pleinement fondé à dire avec $\mathrm{A}$. de Libera que la mystique d'Angèle est porteuse d'un savoir et que ce savoir est " théologiquement articulable $~^{41}$, qu'elle n'est donc pas marginale et ne relève en rien du topos d'un corps jouissant de l'hystérie ${ }^{42}$. En revanche, on ne saurait le suivre lorsqu'il dénie toute pertinence à une vision sexuée de la mystique.

Pourquoi l'historien qui reconnait l'existence d'un corps mystique féminin serait-il condamné à le voir comme "un tissu de symptômes » ${ }^{43}$ ? La mystique féminine n'est pas un pur phantasme historiographique, elle se construit sur les bases du statut social de la femme dans la culture médiévale. Qui peut en douter, sauf à considérer le discours spirituel et théologique comme un système clos et auto-référencé ? Il ne s'agit donc pas de s'arc-bouter sur des "catégorisations "homme" / "femme" dans le champ de la mystique $»^{44}$, mais de délimiter le jeu complexe des polarisations, au féminin et au masculin. Toute la difficulté est de prendre la mesure des champs partagés (et ils sont bien plus étendus en ce qui concerne la mystique féminine qu'on n'a voulu le voir) tout en marquant les limites qui maintiennent des spécificités (qui existent assurément et s'expriment tout particulièrement dans l'appréhension du corps, des sens et des affects).

La subjectivité qui se laisse entrevoir dans la mystique d'Angèle n'est ni l'émanation d'une identité d'essence, ni une simple variation à partir d'un discours théologique, elle résulte d'une construction culturelle mixte qui assigne néanmoins à la femme une place spécifique dans la société. C'est pourquoi, s'il convient de manier avec prudence la catégorie du "mysticisme féminin ", dans la mesure où il s'agit d'un modèle de piété élaboré par l'historiographie mais non théorisé comme tel par les contemporain(e)s, ce serait une amère défaite de la science historique que d'y renoncer tout à fait ${ }^{45}$. 
21 Les phénomènes d'incorporation mystique rapportés dans le Livre permettent de vérifier que, vers 1300 , dans le champ religieux, le corps est devenu un lieu privilégié d'identification du sujet ${ }^{46}$. Cette voie n'est pas spécifiquement féminine, mais elle est amplifiée, exacerbée dans la production mystique du corps féminin. Un corps malléable, poreux, apte aux transformations de forme voire de substance. Car au stade ultime de sa compassion pour les souffrances du Christ, il y a pour Angèle sa transformation en la passion du Christ (165). Le cas d'Angèle met en lumière un moment et une facette d'un processus, inscrit dans la longue durée, de définition d'une subjectivité où l'intériorité de l'être passe par l'affectivité, l'expérience des sens et l'imagination ${ }^{47}$. C'est assurément davantage comme individu in-habité par la divinité ${ }^{48}$ plutôt que comme sujet féminin incorporé que la figure d'Angèle de Foligno se livre à notre regard dans une persistante étrangeté.

\section{BIBLIOGRAPHIE}

AELRED de RIEVAULX, 1961, La Vie de recluse, trad. Ch. Dumont, Paris, Cerf, Sources chrétiennes 76.

Angela da Foligno, terziaria francescana, Atti del Convegno storico nel VII centenario dell'ingresso della beata Angela da Foligno nell'Ordine Francescano Secolare (1291-1991), (Foligno, 17 au 17 novembre 1991), 1992, E. Menestò (dir.), Spolète.

Angèle de Foligno : le dossier, 1999, G. Barone et J. Dalarun (dir.), Rome, École Française de Rome.

BARTOLI Marco et DELVILLE Jean-Pierre, 2005, « Claire d'Assise et Julienne de Cornillon :

mystiques et politiques », in J.-P. Delville (dir.), Mystiques et politiques, Bruxelles, Lumen Vitae, p. 62-79.

BELL Rudolph M., 1994, L'Anorexie sainte. Jeûne et Mysticisme du Moyen Âge à nos jours, Paris, PUF.

BERNARD de CLAIRVAUX, 2003, Sermons sur le Cantique, t. 4, Paris, Cerf, Sources chrétiennes 472.

BOLENS Guillemette, 2000, La Logique du corps articulaire. Les articulations du corps humain dans la littérature occidentale, Rennes, PUR.

BOQUET Damien, 2000, « Le sexe des émotions. Principe féminin et identité affective chez Guerric d'Igny et Aelred de Rievaulx », in P. Henriet et A.-M. Legras (dir.), Au Cloître et dans le monde. Femmes, hommes et sociétés (IXe-XVe siècle), Paris, Presses de l'Université de Paris-Sorbonne, p. 367-378.

-, 2005, L'Ordre de l'affect au Moyen Âge. Autour de l'anthropologie affective d'Aelred de Rievaulx, Caen, Publications du CRAHM.

-, 2006, «Un homme sous influence. Vers une histoire de la personne humaine dans Satan hérétique d'A. Boureau », Cahiers du Centre de Recherches Historiques, 37, p. 189-197.

BOUREAU Alain, 2004, Satan hérétique. Histoire de la démonologie (1280-1330), Paris, Odile Jacob. BOURGAIN Pascale, 1999, « Angèle de Foligno. Le latin du Liber », in Angèle de Foligno : le dossier..., p. 145-167. 
BRUFANI Stefano, 1992, « Angela da Foligno e gli Spirituali », in Angela da Foligno..., p. 83-104.

BYNUM Caroline Walker, 1994, Jeûnes et festins sacrés. Les femmes et la nourriture dans la spiritualité occidentale, Paris, Cerf.

-, 1992, Fragmentations and Redemption. Essays on Gender and the Human Body in Medieval Religion, New York, Zone Books.

CAROZZI Claude, 1976, « Douceline et les autres », in La Religion populaire en Languedoc du XIIIe à la moitié du XIVe siècle, Cahiers de Fanjeaux, n 11, p. 251-267.

CERTEAU Michel de, 2005, Le Lieu de l'autre. Histoire religieuse et mystique, éd. L. Giard, Paris, Gallimard-Le Seuil (coll. Hautes Etudes).

DALARUN Jacques, 1994, Lapsus linguae. La légende de Claire de Rimini, Spolète, Centro italiano di studi sull'alto medioevo.

DREYER Elizabeth, 2000, The Cross in Christian Tradition : From Paul to Bonaventure, New York.

GUERRIC d'IGNY, 1973, Sermons II, trad. dir. par P. Deseille, ", Paris, Cerf, Sources chrétiennes n 202.

GUILLAUME de SAINT-THIERRY, 1953, De la contemplation de Dieu, Paris, Vrin.

IOGNA-PRAT Dominique, 2005, «Édification personnelle et construction ecclésiale », in L'Individu au Moyen Âge, B. M. Bedos-Rezak et D. Iogna-Prat (éd.), Paris, Aubier, p. 247-269.

JACQUES de VITRY 1997, Vie de Marie d'Oignies, trad. J. Miniac, Paris, Actes Sud Babel.

JACQUES de VORAGINE, 2004, La Légende dorée, éd. publiée sous le dir. d'A. Boureau, Paris, Gallimard (coll. Bibliothèque de la Pléiade).

LECLERCQ Jean, 1963, « La dévotion médiévale au crucifié », La Maison Dieu, 75, p. 118-123.

LEONARDI Claudio, 1992, «Angela da Foligno tra teologia e mistica », in Angela da Foligno..., p. 251-259.

LIBERA Alain de, 1991, Penser au Moyen Âge, Paris, Seuil, Points Essais.

-, 1999, « Angèle de Foligno et la mystique « féminine ». Éléments pour une typologie », in Angèle de Foligno : le dossier, G. Barone et J. Dalarun (dir.), Rome, École Française de Rome, p. 345-371.

L'HERMITE-LECLERCQ Paulette, 1996, «La recluse, la femme et l'amour de Dieu », in C. DuhamelAmado et G. Lobrichon (dir.), Georges Duby. L'Ecriture de l'histoire, Bruxelles (Bibliothèque du Moyen Âge, $\mathrm{n}^{\circ}$ 6), p. 379-384.

Il Libro della beata Angela da Foligno, 1985, L. Thier et A. Calufetti (éd.), Grottaferrata, Editiones Collegii S. Bonaventurae ad Claras Aquas. Traduction française de J.-F. Godet sur la base de cette édition, Le Livre d'Angèle de Foligno, d'après les textes originaux, Grenoble, Jérôme Millon, 1995.

MERLO Grado Giovanni, 2006, Au nom de saint François. Histoire des Frères mineurs et du franciscanisme jusqu'au début du XVIe siècle, Paris, Cerf-Editions Franciscaines.

MORSON John, 1978, Christ the Way. The Christology of Guerric of Igny, Kalamazoo, Cistercian Publications.

NAGY Piroska (à paraître), « Larmes et eucharistie. Formes du sacrifice en Occident au Moyen Âge central », in N. Bériou et B. Cazeau (dir.), Pratiques de l'Eucharistie dans les Eglises d'Orient et d'Occident, Paris, Études Augustiniennes.

PAOLI Emore, 1999, « Le due redazioni del Liber : il perché di una riscrittura », in Angèle de Foligno : le dossier..., p. 29-70. 
PETERS Ursula, 1988, Religiöse Erfahrung als literarisches Faktum : zur Vorgeschichte und Genese frauenmystischer Texte des 13. und 14. Jahrhunderts, Tübingen, M. Niemeyer.

RUBIN Miri, 1991, Corpus Christi. The Eucharist in the Late Medieval Culture, Cambridge, Cambridge University Press.

Sainte Claire d'Assise. Documents, 2002, éd. et trad. par D. Vorreux, Les Éditions Franciscaines.

The Life of Christina of Markyate. A Twelfth Century Recluse, 1998 (1ère édition 1983), éd. et trad. par C.H. Talbot, Toronto, University of Toronto Press.

VAUCHEZ André, 1987a, «La sainteté féminine dans le mouvement franciscain », in Les Laïcs au Moyen Âge, Paris, Cerf, p. 189-202.

-, 1987b, « Dévotion eucharistique et union mystique chez les saintes de la fin du Moyen Âge ", in Les Laïcs au Moyen Âge, p. 259-264.

\section{NOTES}

1. Certeau $2005: 328$ (ch. 14 "Mystique ", repris de l'article «Mystique » de l'Encyclopedia universalis, 1985, t. 12, p. 873-878).

2. Voir en ce sens par exemple Carozzi 1976.

3. L'expression est de Libera $1999: 347$.

4. Voir Vauchez 1987b : 189-202.

5. Je laisse de côté ici ce débat très pointu sur l'implication du Livre dans le conflit entre Spirituels et Conventuels. Pour le contexte général, voir Merlo $2006: 203-221$, puis Brufani $1992: 83-104$.

6. Pour l'édition latine, voir Il Libro della beata Angela da Foligno... 1985, et la traduction française sur la base de cette édition, Le Livre d'Angèle de Foligno..., 1995. (Je cite cette traduction avec la référence aux pages dans le corps de l'article pour ne pas alourdir l'appareil de notes.)

7. Sur ce point de la fabrique textuelle du témoignage mystique féminin, voir Peters 1988.

8. Sur ces questions des différentes rédactions du Livre, en latin et en ombrien, et leur ordre chronologique, voir en dernier lieu dans Angèle de Foligno: le dossier 1999 les articles de Paoli : 29-70 et de Bourgain : 145-167.

9. Boureau $2004: 244$ et 246 . L'auteur propose une réflexion fondamentale sur les rapports entre inspiration mystique et subjectivité, p. 227-260.

10. Bartoli et Delville $2005: 62-79$.

11. Bynum 1992: 185. L'ensemble de l'ouvrage offre une excellente mise en perspective des thématiques abordées ici.

12. Sur ce motif de l'ingestion eucharistique, voir Bynum 1992: 341-359 et Vauchez 1987a : 259-264.

13. Voir Bell 1994.

14. Jacques de Vitry $1997: 149$.

15. Jacques de Vitry $1997: 143$.

16. Jacques de Vitry $1997: 81$ et 149-150.

17. Jacques de Vitry $1997: 54$.

18. Voir Nagy (à paraître).

19. Voir par exemple le cistercien du XIIe siècle Guerric d'Igny $1973: 174$.

20. Voir Sainte Claire d'Assise. Documents $2002: 60$. L'épisode est étudié par Bartoli et Delville $2005: 47-62$ 
21. Pour d'autres points de ressemblance entre la Vie de Claire d'Assise par Thomas de Celano et le Livre d'Angèle, voir Dalarun 1994 : 377-378.

22. Voir The Life of Christina of Markyate... 1998: 118.

23. Voir Rubin 1991: 302-306.

24. Voir Boquet $2000: 367-378$.

25. Voir Morson $1978:$ 129-136.

26. Bernard de Clairvaux $2003: 254-255$, « Sermon 61 ».

27. Guerric d'Igny 1973 : 214-215, «Quatrième sermon pour les Rameaux »; voir aussi p. 210-211. Autre référence cistercienne : Guillaume de Saint-Thierry 1953 : 36-37.

28. Aelred de Rievaulx $1961: 140-141$. Pour une analyse de ce qui apparaît comme l'un des exemples les plus précoces de piété mystique proposés aux femmes, voir L'Hermite-Leclercq 1996 : 379-384 et Boquet $2005: 255-274$.

29. Jacques de Voragine 2004 : 363-372 chap. 64 «L'invention de la sainte croix », et 752-759 chap. 131 « L'exaltation de la sainte croix ».

30. Dreyer 2000.

31. Leclercq $1963: 118-123$.

32. Guerric d'Igny 1973 : 183, « Deuxième sermon pour les Rameaux ».

33. Guerric d'Igny $1973: 181$.

34. Guerric d'Igny $1973: 183$.

35. Signum crucis, soit le « signe de croix » mais aussi le « crucifix ».

36. Guerric d'Igny $1973: 187$.

37. Angèle a des images précises sur la forme des clous qu'elle voit sans pointe et carrés, donc plus blessants (199).

38. Voir l'iconographie saisissante sélectionnée par Bynum 1992 : 278-279.

39. Bolens 2000.

40. Je développe ailleurs cet aspect de l'écho de l'anthropologie cistercienne dans le Livre, voir Boquet 2006.

41. Libera 1999 : 366. En revanche, il me paraît trompeur de parler de la "théologie mystique » d'Angèle par référence au traité du Pseudo-Denys qui porte le même titre, voir Dalarun 1994 : 435. La piété mystique d'Angèle transpose des propositions théologiques selon un prisme spirituel qui lui est propre, elle ne formule pas directement une pensée théologique.

42. Voir aussi Leonardi $1992:$ 251-259.

43. Libera $1991: 300$.

44. Libera 1999 : 360 .

45. Voir Libera $1991: 302$.

46. Il est aussi de plus en plus un lieu d'altérité, exposé à la possession par l'autre, comme l'a montré A. Boureau dans son enquête sur la naissance de la démonologie ; voir Boureau 2004.

47. Voir Iogna-Prat $2005: 268-269$.

48. Voir Boureau 2004 : 258, qui parle d'» individu accidentel » à propos d'Angèle.

\section{RÉSUMÉS}

Incorporation mystique et subjectivité féminine d'après le Livre d'Angèle de Foligno. Comment qualifier, dans le cadre d'une histoire de la subjectivité, la piété compassionnelle dont témoigne 
Angèle de Foligno ( $†$ 1309) dans le Mémorial et les Instructions ? En choisissant trois motifs qui déclinent la figure de l'incorporation mystique (dévotions à l'eucharistie, aux plaies du Christ et aux instruments de la Passion), cette étude met au jour les origines monastiques d'une piété fusionnelle et affective, d'abord élaborée dans un environnement spécifiquement masculin aux $\mathrm{XI}^{\mathrm{e}}-\mathrm{XII}{ }^{\mathrm{e}}$ siècles, puis investie par la dévotion des mulieres religiosae (moniales, béguines, recluses ou tertiaires) à partir du XIIIe siècle. Placée dans une perspective de longue durée, la piété d'identification au Christ et à ses souffrances d'Angèle révèle ainsi une sensibilité religieuse aux référents mixtes, masculins et féminins, mais qui a été infléchie par la position sociale des femmes et leurs aspirations propres dans le champ de la piété.

Mystical incorporation and feminine subjectivity in the Book of Angela da Foligno. Within the context of a history of subjectivity, how can we understand the compassionate piety demonstrated by Angela da Foligno $(\dagger$ 1309) in her Memorial and Instructions? Through an analysis of three figures of mystical incorporation (the devotion to the eucharisty, to Christ's wounds and to the instruments of Passion), this study reveals the monastic origins of a fusional and affective piety. It was born in a specifically masculine environment in the 11th and 12th century, and then reappropriated in the devotion of mulieres religiosae (nuns, beguines, recluses or tertiaries) from the 13th century onwards. Considered in a long-term perspective, Angela's piety, which involved identification with Christ and his sufferings, reveals a religious sensibility with mixed gendered references; this sensibility was shaped by women's social condition and by their distinctive aspirations to piety.

\section{INDEX}

Index chronologique : Moyen Âge

Keywords : affective piety, da Foligno Angela, body, eucharisty, feminine mysticism, Middle Ages

Mots-clés : corps, eucharistie, mystique féminine, piété affective, Foligno Angèle (de)

\section{AUTEUR}

\section{DAMIEN BOQUET}

Damien BOQUET est maître de conférences en histoire médiévale à l'université de Provence. Il a publié en 2005 L'ordre de l'affect au Moyen Âge. Autour de l'anthropologie affective d'Aelred de Rievaulx, Caen, Publications du CRAHM et il a participé récemment à l'ouvrage collectif Le Choix de l'homosexualité. Recherches inédites sur la question gay et lesbienne, B. Perreau (dir.), Paris, EPEL, 2007. Il dirige actuellement, avec Piroska Nagy, un programme de l'ANR (Agence Nationale de la Recherche) consacré aux émotions médiévales. 